\title{
Design of OAM Beam Directional Modulation Signal in Communication and Guidance Integration
}

\author{
Changju ZHU, Maozhong SONG, Xiaoyu DANG \\ College of Electronic Information Engineering, Nanjing University of Aeronautics and Astronautics, Jiangjun Ave 29, \\ 211106 Nanjing, China
}

zhu_changju@163.com,smz108@nuaa.edu.cn,dang@nuaa.edu.cn

Submitted November 12, 2019 / Accepted May 5, 2020

\begin{abstract}
An orbital angular momentum directional modulation (OAM-DM) signal is proposed for communication and guidance integration. This signal is transmitted by a uniform circular antenna array (UCA). We divide the array into odd and even antenna groups. Each group is excited by differential coded digital modulation waveform to send different signal constellations in different directions. In order to improve the performance of angle estimation, we have designed specific phase shift sequences to obtain the different OAM modes. Mode detection can eliminate multiple value ambiguity of elevation and azimuth angles. The single antenna receiver can demodulate the OAM-DM signal to communicate, detect OAM modes and estimate angles in different directions. Finally, we assess the effectiveness of the proposed approach via numerical simulation.
\end{abstract}

\section{Keywords}

Directional modulation, signal design, orbital angular momentum

\section{Introduction}

Electromagnetic radiation can carry orbital angular momentum (OAM) [1], [2], and OAM beams of different OAM modes have orthogonality with each other [3]. Due to direction information of OAM beam [4-7], it has potential application in directional modulation (DM) technology. At present, there are some methods to generate OAM beam [8-12]. In the field of radio frequency, UCA has been extensively studied for its flexibility in generating various OAM modes. Researchers have already realized the detection of OAM mode [13-16]. However, there are still fewer detection methods for OAM mode in communication and guidance integration. More recently, application of OAM beam has attracted a lot of interest in radar detection or communication [17-21]. In the face of the increasing threat of a weapon platform and complex electromagnetic environment, the single electronic equipment is not enough to meet the needs of diversified battlefield guidance. Radar communication integration can realize the information and data transmission among wireless devices at the same time of target detection and tracking [22]. At present, there are researches on radar communication integration in which the signal waveform design is an important part of radar communication integration [23]. The paper focuses on the waveform design of dual-function multiple-input-multipleoutput (MIMO) radar communication system and introduces low-complexity algorithms [24]. Considering the similarity of radar and communication in both software and hardware, a co-design integrated waveform of radar and communication based on the multi antenna system is presented in [25]. The optimal combination of the two sub beams and the quantization of the beam form vector that generates the combined beam are investigated in [26]. In [27], a novel multi beam framework using steerable analog antenna arrays, which allows seamless integration of communication and sensing, is studied.

DM can realize the directionality of the signal, ensure the safe transmission of the desired direction signal, and interfere with the constellation of the signal in the unexpected direction [28]. In [28], the DM technology based on phased array needs to take place in the radio frequency stage instead of the conventional baseband modulation, which mainly considers the constellation diagram of the baseband digital modulation signal synthesized in the desired direction. The double beam signal based on DM in [29] is a kind of spatial spread spectrum signal which modulates the spread spectrum sequence and spatial orientation information together. In [30], the baseband terminal synthetic DM signal adopts the artificial noise, which is orthogonal to the desired direction, and the constellation of the signal keeps dynamic change, so that the eavesdropper cannot track the change rule of the constellation of the signal, so it is difficult to track and decode the useful information. The research in [31] is extended to multi beam DM system on the basis of [30]. Therefore, DM technology combined with OAM beam has a special application prospect in radar communication integration.

In this study, we combine directional characteristics of OAM beam radiation pattern with DM technology to produce a new type OAM signal, which can transmit different modulation signal constellation in different directions, for communication and guidance. The single antenna can 
estimate the angle and transmit digital information in radar communication integration.

The remainder of this paper is organized as follows. In Sec. 2, the design idea of combining DM technology with OAM beam is given. Methods of OAM-DM signal design and the demodulation are proposed in Sec. 3. Section 4 introduces the angle estimation method using dual OAM beams. Then, Section 5 evaluates the proposed scheme and analyzes the angle estimation accuracy of different OAM beams. Finally, conclusions are drawn in Sec. 6.

\section{Design of OAM-DM}

The study of OAM beam radiation pattern shows that the direction of OAM beam main lobe will change with the change of OAM mode. Different OAM beams have different directional information. Thus, we combine the DM technology to modulate the OAM mode information into the designed signal. The signal constellation of different OAM modes will be different. Finally, different DM signals are transmitted to different directions.

Figure 1 shows that UCA is used to generate different mode OAM beams. The number of UCA elements is $2 M$, the radius of UCA is $r$, the wavelength is $\lambda$ and the frequency is $f$. Parameters $\delta$ and $\gamma$ represent the elevation and azimuth angle of the receiver, respectively. The azimuth angle of the $m^{\text {th }}$ element is $\gamma_{m}$. The phase difference $\varphi$ between excitation signals of adjacent elements in UCA can be written as [32]

$$
\varphi=\frac{\pi l}{M}
$$

where $l$ is the mode of the OAM beam, $M$ is half the number of UCA elements. In theory, the mode of the OAM can be an arbitrary real number. The relationship between the generating mode and the number of antenna array elements can be obtained by [33].

$$
|l|_{\max }<M \text {. }
$$

The range of phase difference between adjacent elements satisfies $\varphi \in(-\pi, \pi)$. We design two sets of excitation signals for the odd and even array elements in UCA. Then, the two groups of signals are coded to modulate the phase difference $\varphi$ between odd and even array elements into the signal. The modulation method can connect the phase difference $\varphi$ with the quaternary digital information to complete the communication. The receiver can get the mode value of OAM beam by demodulating the phase difference $\varphi$, and then get the OAM beam pattern information to estimate the angle. In this process, the basic UCA information, which the receiver needs, will be transmitted by communication.

The design of DM signal can be used for both communication and angle estimation. This modulation method also provides a new thinking for OAM mode detection.

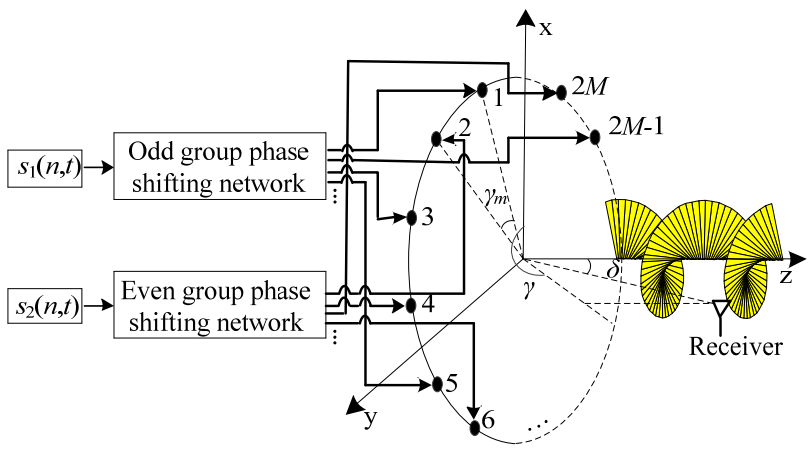

Fig. 1. Diagram of transmission OAM-DM signal.

\section{Modulation and Demodulation of Signal}

\subsection{Signal Modulation}

In this paper, both elevation angle and azimuth angle estimations of the target by dual OAM beams are closely related to the OAM mode. The receiver can estimate the elevation and azimuth as long as the mode of OAM beam is detected. The designed OAM-DM signal contains OAM mode information.

As shown in Fig. 1, antennas in UCA are divided into odd and even group. Then, the odd array elements are excited by $s_{1}(n, t)=c_{11}(n) \cos \omega t+c_{12}(n) \sin \omega t$ and phase shift sequence $\left\{\exp \left(\mathrm{j} l \gamma_{1}\right), \exp \left(\mathrm{j} l \gamma_{3}\right), \ldots, \exp \left(\mathrm{j} l \gamma_{2 M-1}\right)\right\} \quad$ respectively, while the even array elements are excited by $s_{2}(n, t)=c_{21}(n) \cos \omega t+c_{22}(n) \sin \omega t$ and phase shift sequence $\left\{\exp \left(\mathrm{j} l \gamma_{2}\right), \exp \left(\mathrm{j} l \gamma_{4}\right), \ldots, \exp \left(\mathrm{j} l \gamma_{2 M}\right)\right\} \quad$ respectively. Where, $\omega$ is the carrier angular frequency of the transmitting signal, $c_{i j} \in[-1,0,1](i, j=1,2)$ is the element of the encoding matrix, $\gamma_{2 m-1}$ and $\gamma_{2 m}$ are azimuth angles of the antenna elements in UCA. The received signal $r(n, t, m)$ can be written by

$$
\begin{aligned}
& r(n, t, m)= \\
& {\left[s_{1}(n, t) s_{2}(n, t)\right]\left[\begin{array}{l}
g(1,2 m-1) \exp [-\mathrm{j} \varphi(1,2 m-1)] \\
g(2,2 m) \exp [-\mathrm{j} \varphi(2,2 m)]
\end{array}\right]} \\
& =\left[\begin{array}{ll}
a_{\mathrm{I}}(n) & a_{\mathrm{Q}}(n)
\end{array}\right]\left[\begin{array}{c}
\cos [\omega t-\varphi(1,2 m-1)] \\
\sin [\omega t-\varphi(1,2 m-1)]
\end{array}\right], \\
& \left\{\begin{array}{l}
g(1,2 m-1)=\exp \left[\mathrm{j} k r \sin \delta \cos \left(\gamma-\gamma_{2 m-1}\right)\right], \\
g(2,2 m)=\exp \left[\mathrm{j} k r \sin \delta \cos \left(\gamma-\gamma_{2 m}\right)\right], \\
\varphi(1,2 m-1)=l \gamma_{2 m-1}, \\
\varphi(2,2 m)=l \gamma_{2 m}
\end{array}\right.
\end{aligned}
$$

where $m$ represents the $m^{\text {th }}$ element, $k=2 \pi / \lambda, \delta$ is the elevation angle, $\gamma$ is the azimuth angle, $a_{\mathrm{I}}(n)$ and $a_{\mathrm{Q}}(n)$ are equivalent baseband signals. The equivalent complex baseband signal $a(n)$ can be expressed as 


$$
\begin{aligned}
& a(n)=a_{\mathrm{I}}(n)+\mathrm{j} a_{\mathrm{Q}}(n), \\
& \left\{\begin{aligned}
a_{\mathrm{I}}(n)= & c_{11}(n) g(1,2 m-1)+c_{21}(n) g(2,2 m) \cos \varphi \\
& +c_{22}(n) g(2,2 m) \sin \varphi, \\
a_{\mathrm{Q}}(n)= & c_{12}(n) g(1,2 m-1)-c_{21}(n) g(2,2 m) \sin \varphi \\
& +c_{22}(n) g(2,2 m) \cos \varphi
\end{aligned}\right.
\end{aligned}
$$

where $\varphi=\varphi(2,2 m)-\varphi(1,2 m-1)$. The matrix $\mathbf{K}$ can be obtained from equivalent complex baseband signal $a(n)$.

$$
\mathbf{K}=[g(1,2 m-1) g(2,2 m) \cos \varphi g(2,2 m) \sin \varphi]^{\mathrm{T}} .
$$

The transmit signal is divided into $n$ frames, and the $n^{\text {th }}$ frame has two chirps which are expressed by

$$
\mathbf{A}(n)=\left[a_{\mathrm{I}}(1, n) a_{\mathrm{Q}}(1, n) a_{\mathrm{I}}(2, n) a_{\mathrm{Q}}(2, n)\right]^{\mathrm{T}} .
$$

Design the modulation matrix $\mathbf{P}(n)$ of differential coding to obtain the differential iteration relation.

$$
\mathbf{P}(n)=\left(\mathbf{G}^{z_{n}}\right)^{\mathrm{T}} \mathbf{P}(n-1)
$$

where $z_{n}$ is the data sequence to be transmitted, $z_{n}=0,1,2,3$. $\mathbf{P}(1)$ is an initialization matrix.

$$
\mathbf{P}(1)=\left[\begin{array}{rrr}
1 & 1 & 0 \\
0 & 0 & 1 \\
-1 & 1 & 0 \\
0 & 0 & 1
\end{array}\right], \mathbf{G}=\left[\begin{array}{cccc}
0 & 1 & 0 & 0 \\
-1 & 0 & 0 & 0 \\
0 & 0 & 0 & 1 \\
0 & 0 & -1 & 0
\end{array}\right] .
$$

The iterative relation of the modulation matrix $\mathbf{A}(n)$ is obtained by

$$
\mathbf{A}(n)=\mathbf{P}(n) \mathbf{K}
$$

After coding and modulation, the signal when transmitting data $z_{n}$ in the $n^{\text {th }}$ frame can be expressed as

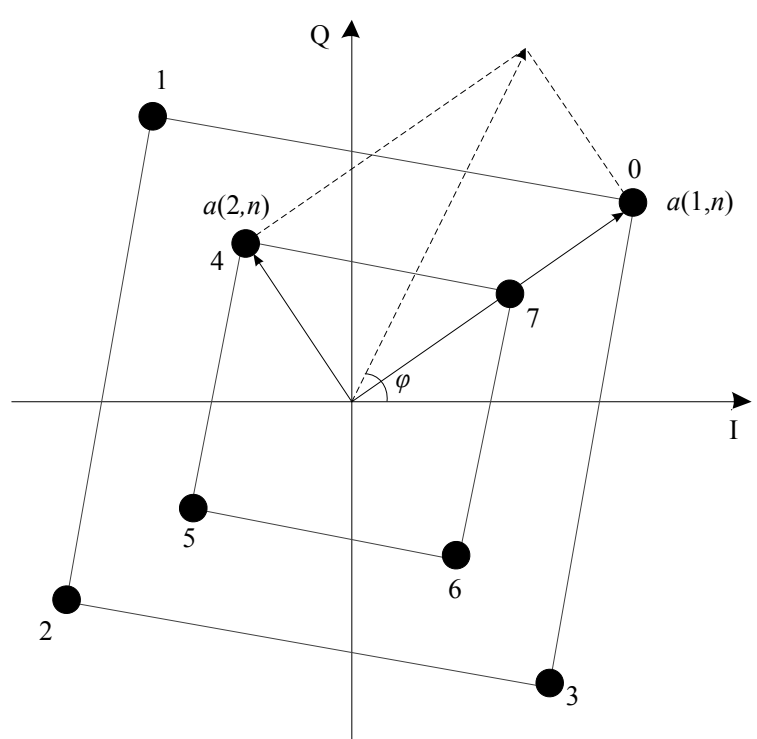

Fig. 2. The modulation relationship of the spatial parameters.

$$
\mathbf{A}(n)=\left\{\begin{array}{l}
{\left[\begin{array}{l}
g(1,2 m-1)+g(2,2 m) \cos (\varphi) \\
g(2,2 m) \sin (\varphi) \\
-g(1,2 m-1)+g(2,2 m) \cos (\varphi) \\
g(2,2 m) \sin (\varphi)
\end{array}\right], z_{n}=0} \\
{\left[\begin{array}{l}
-g(2,2 m) \sin (\varphi) \\
g(1,2 m-1)+g(2,2 m) \cos (\varphi) \\
-g(2,2 m) \sin (\varphi) \\
-g(1,2 m-1)+g(2,2 m) \cos (\varphi)
\end{array}\right], z_{n}=1} \\
-g(1,2 m-1)-g(2,2 m) \cos (\varphi) \\
-g(2,2 m) \sin (\varphi) \\
g(1,2 m-1)-g(2,2 m) \cos (\varphi) \\
-g(2,2 m) \sin (\varphi)
\end{array}\right], z_{n}=2
$$

Figure 2 shows the signal constellation and the modulation relationship of the spatial parameters. The constellation will be distorted with the change of OAM mode. The relationship among two QPSK (Quadrature Phase Shift Keying) signals and the phase difference $\varphi$ can be seen from the constellation.

The equations of spatial modulation are

$$
\begin{gathered}
a(1, n)-a(2, n)=2 g(1,2 m-1), \\
a(1, n)+a(2, n)=2 g(2,2 m)(\cos \varphi+\mathrm{j} \sin \varphi)
\end{gathered}
$$

where $a(i, n)=a_{\mathrm{I}}(i, n)+\mathrm{j} a_{\mathrm{Q}}(i, n)$ is the equivalent complex signal $(i=1,2)$. From (11) and (12), it is obvious that the phase difference $\varphi$ equals to the angle between $a(1, n)-a(2, n)$ and $a(1, n)+a(2, n)$.

\subsection{Signal Demodulation}

Due to the distortion of the OAM-DM signal constellation, the receiver is not suitable for the conventional QPSK signal demodulation method to demodulate OAMDM signal, which can improve the communication security performance for communication and guidance integration. Therefore, we propose a differential modulation method of OAM-DM signal.

The differential equation of the received signal phase can be expressed as

$$
\phi(i, n)=\phi(i, n-1)+\frac{\pi z_{n}}{2}, i=1,2 .
$$

The microwave field from the transmitter to receiver will be introduced a delayed phase $\phi$ and an amplitude 
attenuation $\rho$. Then the received signal $V(i, n)$ in the equivalent baseband form is written as

$$
V(i, n)=\rho a(i, n) \exp (-\mathrm{j} \phi) .
$$

The carrier phase $\phi$ in the receiver can be derived by the following equation.

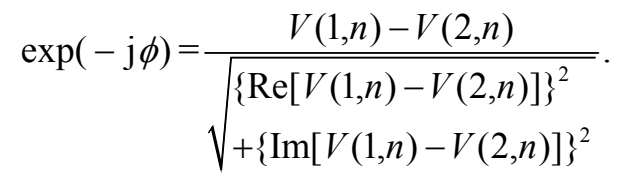

The differential demodulation relation of the received signal $V(i, n)$ is given by

$$
V(i, n)=V(i, n-1) \exp \left(\mathrm{j} \frac{z_{n} \pi}{2}\right)
$$

where $i=1,2$. The data sequence $z_{n}$ can be calculated based on the theory of maximum likelihood estimation.

$$
\exp \left(\mathrm{j} \frac{\hat{\pi} z_{n}}{2}\right)=\frac{\left[\begin{array}{l}
V(1, n) V^{*}(1, n-1) \\
+V(2, n) V^{*}(2, n-1)
\end{array}\right]}{\left[\begin{array}{l}
V(1, n-1) V^{*}(1, n-1) \\
+V(2, n-1) V^{*}(2, n-1)
\end{array}\right]} .
$$

The digital information demodulated by (17) indicates that the receiver can receive the digital information of the transmitter. Due to the existence of positive and negative values of the OAM mode values, it is necessary to distinguish positive and negative values for the demodulation phase difference $\varphi$. Therefore, the phase difference can be demodulated by arcsine function. The phase difference $\varphi$ between odd and even group of UCA is obtained by

$$
\varphi^{\prime}=\arcsin \frac{\operatorname{Im}[V(1, n)+V(2, n)]}{\sqrt{[V(1, n)+V(2, n)][V(1, n)+V(2, n)]^{*}}} .
$$

The mode value of OAM beam can be calculated by

$$
l^{\prime}=\frac{M \varphi^{\prime}}{\pi} .
$$

The estimating phase difference $\varphi^{\prime}$ exists the multiple values in (18). And the relationship between the number of antennas in UCA and the OAM mode is $l \in[-M / 2, M]$. The recognition range of $\varphi^{\prime}$ is $[-\pi / 2, \pi]$. The phase difference $\varphi$ should be $[-\pi / 2, \pi)$. So the range of demodulation helical phase difference $\varphi^{\prime}$ is also $[-\pi / 2, \pi)$. In this paper, we only use eight specific modes of OAM beams. Therefore, the demodulated phase can satisfy the needs of this study.

The parameters $g(1,2 m-1)$ and $g(2,2 m)$ can be calculated by

$$
\begin{gathered}
\hat{g}(1,2 m-1)=\frac{V(1, n)-V(2, n)}{2}, \\
\hat{g}(2,2 m)=\frac{V(1, n)+V(2, n)}{2} \exp \left(-\mathrm{j} \varphi^{\prime}\right) .
\end{gathered}
$$

In fact, when UCA generates OAM beam, $g(1,2 m-1)$ $=g(2,2 m)$. Thus, the radiation pattern $g_{l}$ of OAM beam can be obtained by

$$
\begin{aligned}
g_{l} & =\sum_{m=1}^{M}\left[\begin{array}{c}
\hat{g}(1,2 m-1) \exp \left(-\mathrm{j} l^{\prime} \frac{2 m-1}{M} \pi\right) \\
\hat{g}(2,2 m) \exp \left(-\mathrm{j} l^{\prime} \frac{2 m}{M} \pi\right)
\end{array}\right] \\
& =\sum_{m=1}^{2 M} \frac{\hat{g}(1,2 m-1)+\hat{g}(2,2 m)}{2} \exp \left(-\mathrm{j} l^{\prime} \frac{m}{M} \pi\right) .
\end{aligned}
$$

The transmitter sends the necessary parameters of UCA to the receiver, which demodulates the pattern information of OAM beam. In summary, we can use the single receiver to detect the OAM mode, the radiation pattern and the propagation phase. Thus, the estimation of elevation angle and azimuth angle can be completed. The receiver only needs to receive the information of the transmitter, and does not need to return the information to the transmitter.

\section{Angle Estimation of Single Receiving Antenna}

\subsection{Elevation Angle Estimation}

The radiation pattern is independent of azimuth and symmetric around the beam axis. According to the property of Bessel function, the intensity is null along the beam axis when $l \neq 0$. The angle of maximum gain is determined by wavenumber, array radius, and OAM mode. When the array parameters are fixed, the angle of maximum gain will change with OAM modes.

Figure 3 plots the elevation profile of the radiation pattern of eight OAM beams with $M=25, r=0.05 \mathrm{~m}$ and $f=9 \mathrm{GHz}$. For each mode, the lobe near beam axis possesses maximum gain, called main lobe. The radiation intensity of a single mode OAM beam corresponds to multiple elevation angles. Thus, it is not easy to locate the target in the entire main-lobe region no matter what the elevation angle is. However, elevation angle ranges of different OAM beams exist intersection region. OAM beams of two adjacent mode values can eliminate elevation multiple values.

Elevation angle-measuring ranges of eight OAM beams are as follows (Sampling interval is $0.05^{\circ}$ ). $|l|=1$ : $\left[5.50^{\circ}, 17.00^{\circ}\right] ;|l|=2:\left[11.75^{\circ}, 25.20^{\circ}\right] ;|l|=3:\left[17.9^{\circ}\right.$, $\left.33.35^{\circ}\right] ;|l|=4:\left[24.1^{\circ}, 41.8^{\circ}\right] ;|l|=5:\left[30.35^{\circ}, 50.8^{\circ}\right] ;|l|=6$ : $\left[36.55^{\circ}, 60.2^{\circ}\right] ;|l|=7:\left[42.5^{\circ}, 68.6^{\circ}\right] ;|l|=8:\left[47.75^{\circ}\right.$, $\left.74.05^{\circ}\right]$. We use the radiation intensity of dual-OAM beam to map the elevation angle. OAM mode detection can lock the mapping in a certain range to eliminate the influence of other OAM beams on elevation angle measurement.

Elevation angle estimation ranges of each two adjacent OAM modes are as follows. $|l|=1,2$ : $\left[11.75^{\circ}\right.$, $\left.17.00^{\circ}\right],|l|=2,3:\left[17.9^{\circ}, 25.2^{\circ}\right],|l|=3,4:\left[24.1^{\circ}, 33.35^{\circ}\right]$, 


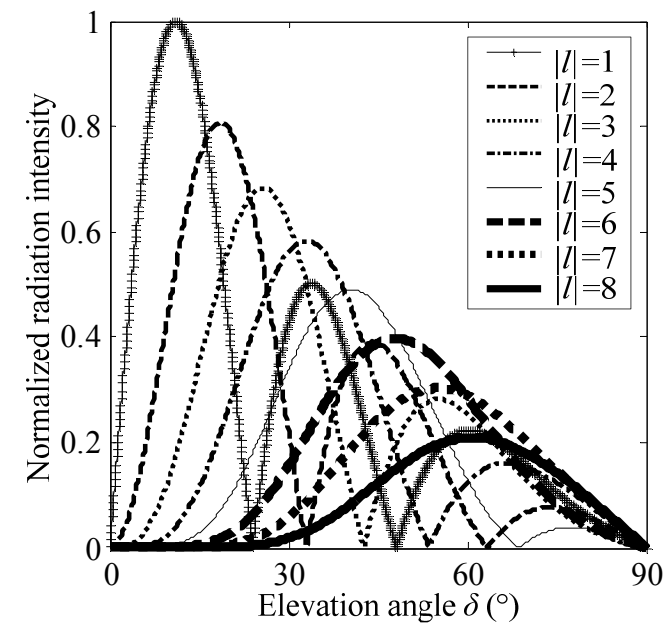

Fig. 3. Elevation profile of OAM radiation pattern: the angle of maximum gain increases with OAM mode $l \mid$.

$|l|=4,5:\left[30.35^{\circ}, 41.8^{\circ}\right],|l|=5,6:\left[36.55^{\circ}, 50.8^{\circ}\right],|l|=6,7:$ $\left[42.5^{\circ}, 60.2^{\circ}\right],|l|=7,8:\left[47.75^{\circ}, 68.6^{\circ}\right]$. We use the radiation intensity ratio of OAM beams to map the elevation angle.

According to radiation patterns of OAM beams in Fig. 3 and [19], OAM beam width increases with the increase of the mode, which makes the range of angle measurement larger. However, the radiation intensity will decrease, which will affect the accuracy of angle measurement. The application of large mode OAM beam is a difficulty in the future research. Therefore, eight kinds of OAM beams are properly selected for the study of communication guidance integration, instead of more OAM modes.

\subsection{Azimuth Angle Estimation}

The phase front of the OAM beam is related to the OAM mode value. The phase varies by $2 \pi l$ rad in a wavelength. The phase of the OAM beam can be correlated with the azimuth of the receiver by the wavelength $\lambda$ and propagation distance $D$. The intensity patterns have a singularity in the center of the main beam [34]. We derive the phase propagating characteristics of OAM beam with distance $D$ which can be written as

$$
\left.\psi_{l}=\frac{2 \pi l}{\lambda}\left(D-\frac{\lambda}{|l|} \mid \frac{D|l|}{\lambda}\right\rfloor\right) .
$$

Without losing generality, take $l=1$ as an example. The phase of the detected OAM beam at the receiver is the azimuth of the receiver when $l=1$ and $d=\alpha \lambda(\alpha=0,1$, $2, \ldots)$. Therefore, when $\alpha$ is not an integer, the azimuth angle of the receiver needs an angle compensation value. It can avoid measuring the propagation distance $D$ that the phase difference between dual OAM beams at the receiver is mapped to the phase of dual OAM beams. When $l=1$, the propagation phase of the OAM beam received by the receiver is the azimuth angle of the receiver. When $l=1$, $2, \ldots,|l|_{\max }$, the phase changes $2 \pi l \mathrm{rad}$ in a wavelength. In Fig. 4, dotted lines represent the phase propagation characteristics of different modes. In a wavelength, the relation- ship between phase propagation and distance can be expressed as a piecewise function, which can be written as

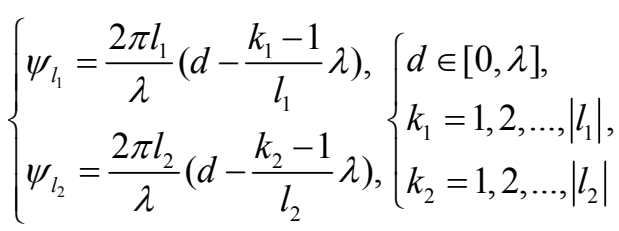

where $k$ is the $k^{\text {th }}$ period in a wavelength. Without losing generality, we set the OAM mode of each group as $l_{2}>l_{1}$, then $l_{2}=l_{1}+1$. As shown in Fig. 4 , the phase propagation of OAM beam varies periodically with the propagation distance in a wavelength, and the number of periods is equal to the mode value. Write (24) as

$$
\Delta \psi=\psi_{l_{2}}-\psi_{l_{1}} .
$$

In Fig. 4, the solid line is obtained by the propagation phase difference between two OAM beams in a wavelength, and the dotted line indicates that the propagation phase of OAM beam is periodically changing, with the range of $[0,2 \pi]$ and the number of periods of phase change is $|l|$ in a wavelength. From these changes, it is deduced that there is no multi-value of the propagation phase difference between two OAM beams with modes $l_{1}$ and $l_{2}$ in a wavelength. According to the phase difference subsection curve in Fig. 4(a)-(f), in a wavelength, there is always a one-toone correspondence between the propagation phase difference and the propagation distance of two OAM beams with modes $l_{1}$ and $l_{2}$, and the propagation phase difference function can be divided into $2 l_{1}$ segments. Thus, the interval of the phase difference $\Delta \psi$ in a wavelength is divided into $2 l_{1}$, $i=1,2, \ldots, 2 \times \min \left\{l_{1}, l_{2}\right\}, i$ is the $i^{\text {th }}$ interval. The relationship between the propagation distance $d_{i}$ and $\Delta \psi$ in a wavelength is

$$
\left\{\begin{array}{l}
d_{2 i-1}=\frac{\Delta \psi}{2 \pi\left(l_{2}-l_{1}\right)} \lambda \\
d_{2 i}=\frac{\Delta \psi-2 \pi}{2 \pi\left(l_{2}-l_{1}\right)} \lambda
\end{array}, d_{i} \in\left[\frac{(2 i-2) \lambda}{l_{2}}, \frac{(2 i-1) \lambda}{l_{1}}\right]_{i} .\right.
$$

Therefore, the receiver detects the propagation phase of OAM beam to map the distance $d$ in a wavelength, which can avoid directly measuring the propagation distance $D$. The value range of distance $d$ is $[(\alpha-1), \alpha \lambda], \alpha$ represents the $\alpha^{\text {th }}$ wavelength. In this way, we can obtain the azimuth compensation value. The compensation value of azimuth angle can be obtained by

$$
\Delta \gamma=\frac{2 \pi l}{\lambda} d,\left\{\begin{array}{l}
d \in[(\alpha-1) \lambda, \alpha \lambda], \\
\alpha=1,2,3, \ldots
\end{array} .\right.
$$

The number of azimuth multiple values is equal to the mode value. Multiple azimuth angles can be expressed as

$$
\gamma_{l}^{h}=\frac{\psi_{l}+2 \pi(|l|-h)}{l}-\Delta \gamma, h \in[1,|l|]
$$

where $h$ denotes the $h^{\text {th }}$ multi-value. We still use dual beams to eliminate multiple values. 


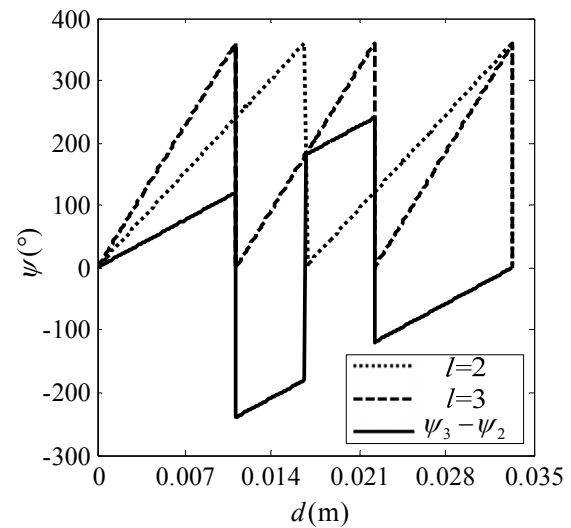

(a)

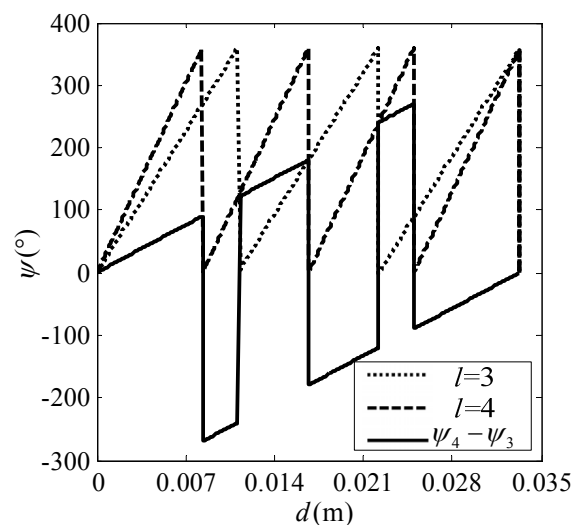

(b)

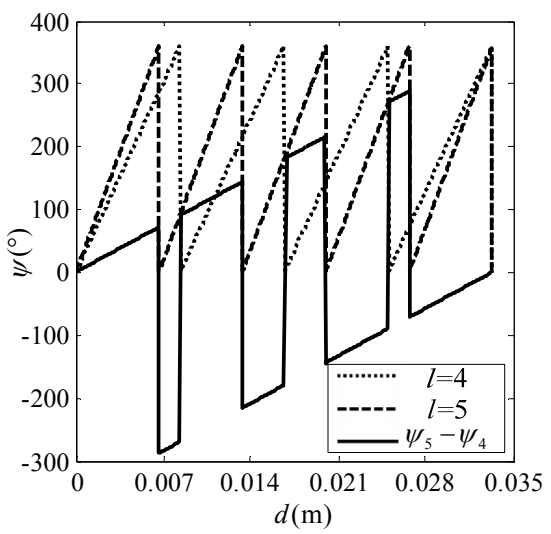

(c)

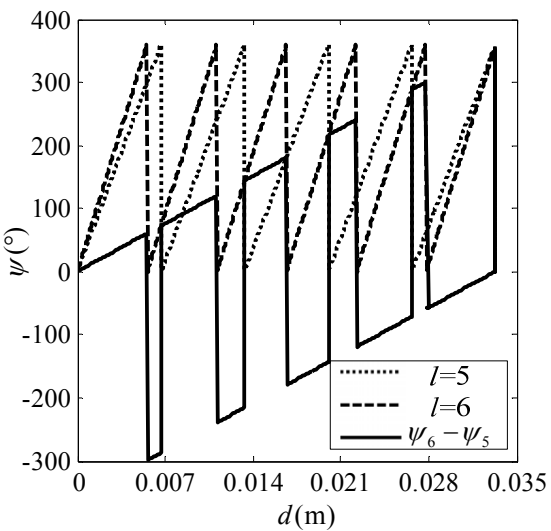

(d)

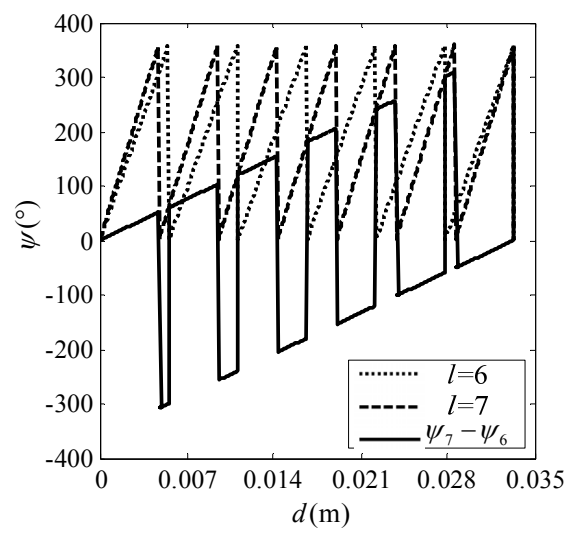

(e)

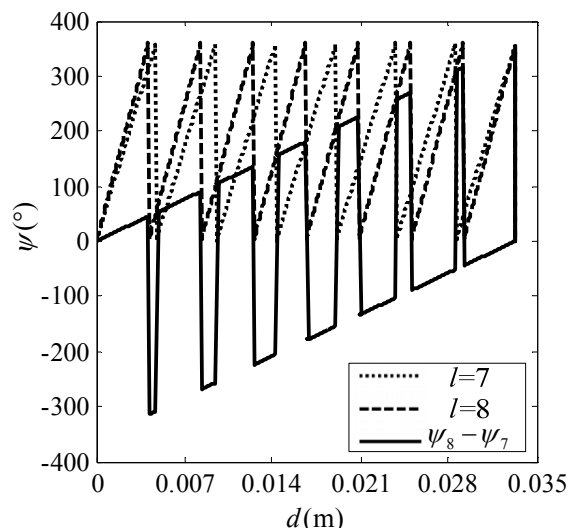

(f)

Fig. 4. The mapping relationship between propagation phase difference and azimuth compensation in a wavelength $(\lambda=1 / 30 \mathrm{~m})$.

\section{Simulation Results and Discussion}

In this section, we simulate the communication bit error rate (BER), OAM mode detection and angles estimation of the scheme on MATLAB platform. In the simulation, 1000 OAM-DM signals are randomly generated under different SNRs.

$S N R=\left(E_{\mathrm{b}} / N_{0}\right) \times\left(R_{\mathrm{b}} / W\right)$, where $E_{\mathrm{b}}$ denotes the energy per unit bit, $N_{0}$ denotes the noise power spectral density, $R_{\mathrm{b}}$ is the bit transmission rate, $W$ is the equivalent noise bandwidth of the receiver. $W$ changes with the modulation mode. When QPSK is adopted, $W$ is $1 \times R_{\mathrm{b}}$. When BPSK modulation is adopted, $W$ is $2 \times R_{\mathrm{b}}$, then $S N R=E_{b} /\left(N_{0} / 2\right)$. In this paper, QPSK modulation is adopted. Therefore, $S N R=E_{\mathrm{b}} / N_{0}$.

Figure 5 shows the BER performance in Additive White Gaussian Noise (AWGN) channel. The BER decreases with the increase of SNR. We choose the higher SNR for the best guidance performance test of OAM-DM signal. In following simulations, 1000 OAM-DM signals are generated under SNR of $15 \mathrm{~dB}$. The phase difference $\varphi$ and OAM mode, which receiver demodulates, are given in Tab. 1 and Tab. 2. Detecting the mode of OAM beam at receiver is the key factor in elevation and azimuth angle estimation. 


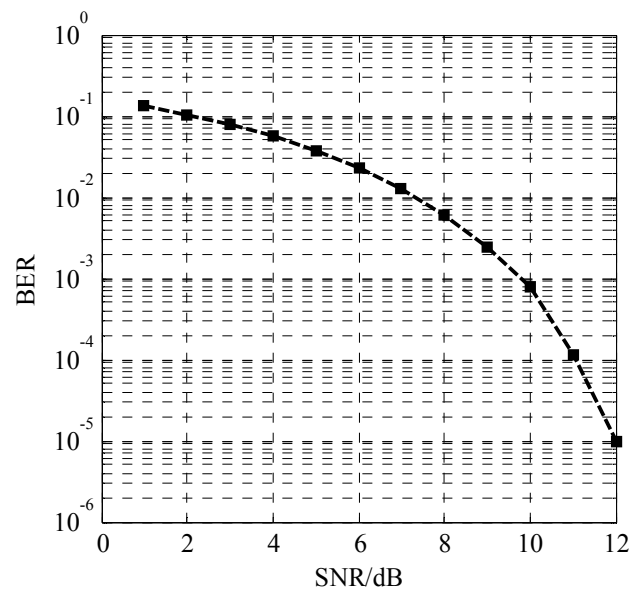

Fig. 5. Performance of the bit error rate in AWGN channel.

\begin{tabular}{|c|c|c|c|c|c|c|c|c|}
\hline$\varphi\left(^{\circ}\right)$ & 7.20 & 14.40 & 21.60 & 28.80 & 36.00 & 43.20 & 50.40 & 57.60 \\
\hline$\varphi^{\prime}\left({ }^{\circ}\right)$ & 7.26 & 14.70 & 21.59 & 28.79 & 36.52 & 43.39 & 50.50 & 57.58 \\
\hline
\end{tabular}

Tab. 1. Modulation phase $\varphi$ and demodulation phase $\varphi^{\prime}$.

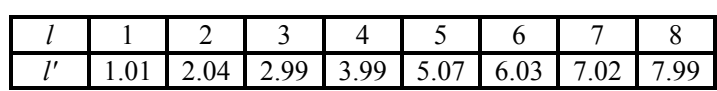

Tab. 2. Emission mode $l$ and detection mode $l^{\prime}$.

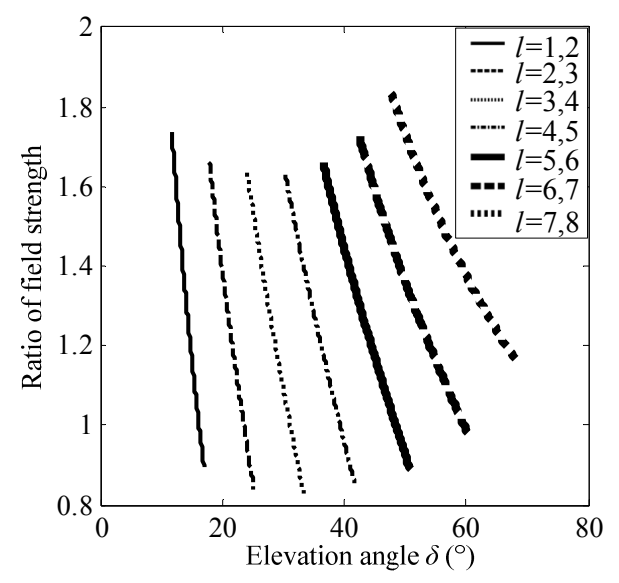

Fig. 6. Elevation angle mapped by ratio of radiation intensity.

As shown in Fig. 6, in a monotonic interval, the ratio and elevation angle always correspond one to one. The higher the slope of the elevation angle estimation curve, the higher the sensitivity. However, the monotone interval of the curve is intersected, which makes the angle estimation appear multiple values. We can use OAM mode detection to distinguish multiple values.

Essentially, the positive and negative values of OAM beam modes have no effect on the elevation angle estimation. The root mean square error (RMSE) of elevation angle is shown in Tab. 3. However, the larger the mode value $|l|$ of OAM beam is, the larger the width of the main lobe will be, which will reduce the sensitivity of elevation angle estimation and lead to the increase of elevation angle estimation error. Therefore, the OAM beam with large mode can be used for rough estimation and the OAM beam with small mode can be used for precise estimation.
Figure 7 plots multivalued curves of different modes. Multiple azimuth angles can always map an azimuth angle $\gamma$. For the estimation of azimuth angle, mode values of OAM beams are equal sign or negative sign. For the convenience of azimuth angle estimation, the mode values of dual-OAM beam should be taken as a positive sign or a negative sign at the same time. The azimuth angle corresponding to each curve can be also distinguished by mode detection. As shown in Fig. 7(a)-(f), when the receiver only receives the propagation phase of one OAM beam, no

\begin{tabular}{|c|c|c|c|c|c|c|c|}
\hline$l$ & 1,2 & 2,3 & 3,4 & 4,5 & 5,6 & 6,7 & 7,8 \\
\hline RMSE $\left.^{\circ}\right)$ & 0.07 & 0.08 & 0.15 & 0.18 & 0.30 & 0.52 & 1.02 \\
\hline
\end{tabular}

Tab. 3. RMSE of elevation angle.

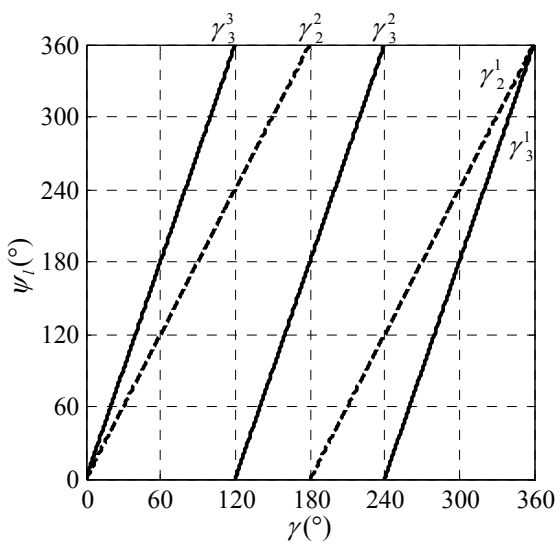

(a)

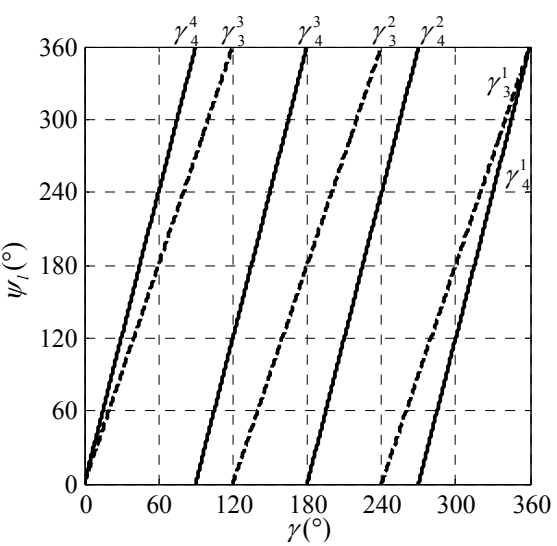

(b)

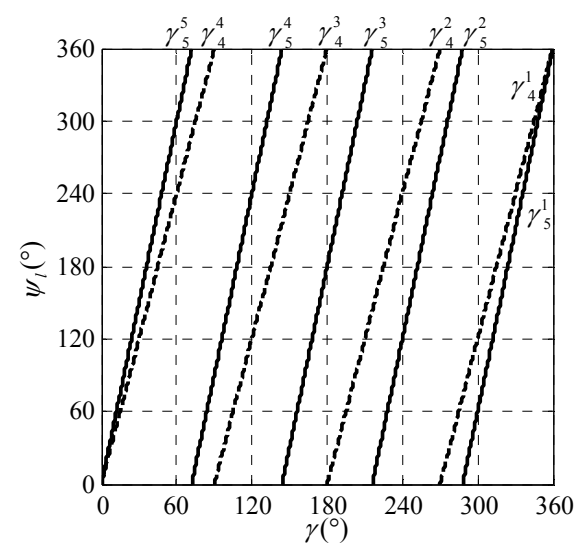

(c) 


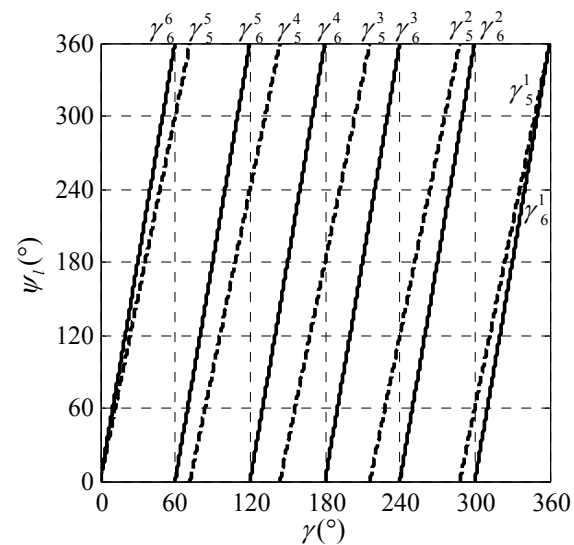

(d)

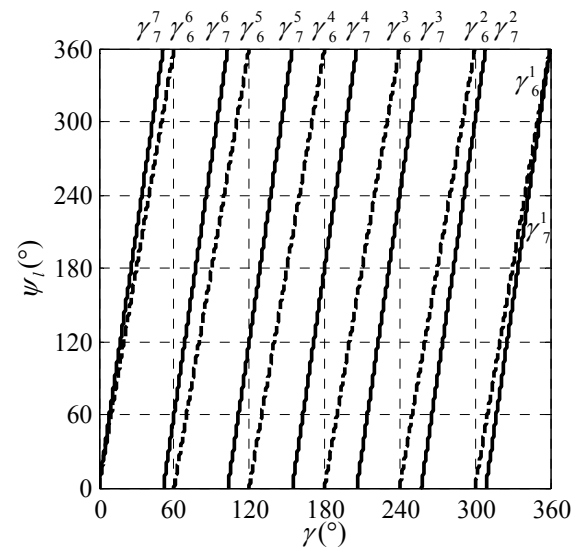

(e)

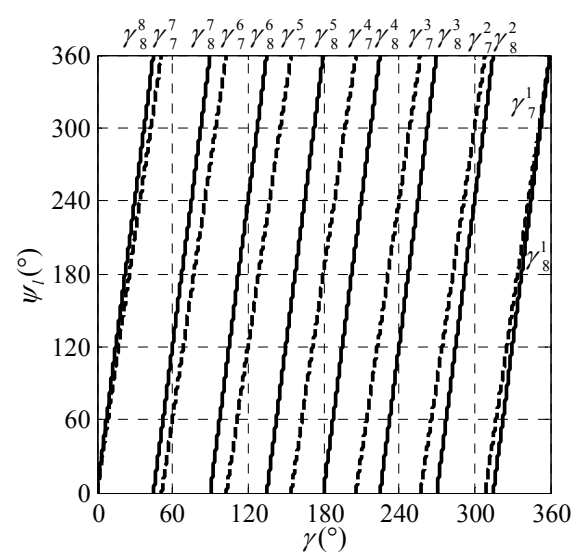

(f)

Fig. 7. The mapping relationship between azimuth angle multi-value and azimuth angle

\begin{tabular}{|c|c|c|c|c|c|c|c|}
\hline$l$ & 1 & 2,3 & 3,4 & 4,5 & 5,6 & 6,7 & 7,8 \\
\hline $\left.\operatorname{RMSE~}^{\circ}\right)$ & 0.02 & 0.03 & 0.03 & 0.03 & 0.04 & 0.06 & 0.09 \\
\hline
\end{tabular}

Tab. 4. RMSE of azimuth angle.

matter whether the mode value is detected or not, the mapped azimuth angle has multiple values and the larger the mode, the more the number of multiple values. When the receiver detects propagation phases of different dualOAM beams at an azimuth angle, it will also map multiple azimuth angles, but the number of multiple values is relatively reduced. For example, when the receiver detects that propagation phases of dual OAM beams are $120^{\circ}$ and $180^{\circ}$, the azimuth angle mapped by OAM beams, which mode values are 2 and 3 in Fig. 7(a), is $60^{\circ}$, while the azimuth angle mapped by OAM beam mode values of 3 and 4 in Fig. $7(\mathrm{~b})$ is $300^{\circ}$. However, the receiver is only located in one of $60^{\circ}$ and $300^{\circ}$ directions. From Fig. 7(a) to (f), there are many cases similar to this kind of azimuth ambiguity. When the receiver detects modes of dual-OAM beams, it can determine the azimuth angle in a set of mapping curve to get the unique azimuth angle.

Table 4 shows that the larger the mode of OAM beam is, the larger is the estimation error of azimuth angle, which is affected by the phase singularity point. Azimuth estimation accuracy of OAM beams with smaller modes is better. This is because the larger the OAM beam mode is, the larger the singular region of the phase center is. Thus, the estimation of OAM propagation phase $\psi_{l}$ will be affected. Moreover, the OAM beam with a larger mode will have larger error in azimuth angle estimation.

Several researchers stressed the fact that the particular spatial profile of OAM beams could be used to perform radar and satellite localization operations [35]. In this paper OAM-DM signals generated by UCA are studied, which provides a reference scheme for the application of OAM beam in the future radar guidance and 6G integration in the RF (Radio Frequency) field, and the scheme is simulated in AWGN channel. Distortion characteristics of OAM beams in different channels are still in the research stage [35]. Therefore, the optimization of OAM-DM signal in other channels needs to be further studied in the future work. At present, there are more researches on the application of OAM beam, and more OAM modes are also being studied. However, related work mainly studies the communication $[10,14,20,21]$ and detection [17-19] of OAM beam independently. OAM-DM signal in this study integrates communication and guidance together, which provides a feasible method for a single device to realize multi-function.

\section{Conclusion}

In this paper, the design scheme of DM signal combined with OAM beam is proposed. The phase difference between adjacent elements of UCA which can generate OAM beams is modulated into the signal. Depending on the directivity of the OAM beam main lobe, modulation signals can send different constellations in different directions. Distortion degree of the constellation transmitted by the modulation signal in different directions is different. Due to the distortion of the constellation, it is sometimes difficult to demodulate QPSK signal by conventional methods. Thus, we design a method using a single antenna to demodulate DM signal, which can improve the security of information transmission to a certain extent. The scheme is suitable for using micro receiver to achieve communication and angle estimation in radar communication integration. 


\section{Acknowledgments}

This work was supported by the National Natural Science Foundation of China under grant NSFC61571224 and the National Key Scientific Instrument and Equipment Development Projects of China under grant 2013YQ200607. The authors would also like to acknowledge the anonymous reviewers for their helpful comments.

\section{References}

[1] VERBEECK, J., TIAN, H., SCHATTSCHNEIDER, P. Production and application of electron vortex beams. Nature, 2010, vol. 467, no. 7313 , p. 301-304. DOI: $10.1038 /$ nature09366

[2] URIBE-PATARroyo, N., FrAinE, A., SiMON, D. S., et al. Object identification using correlated orbital angular momentum states. Physical Review Letters, 2012, vol. 110, no. 4, p. 1-5. DOI: 10.1103/PhysRevLett.110.043601

[3] WANG, Z., ZHANG, N., YUAN, X. C., High-volume optical vortex multiplexing and de-multiplexing for free-space optical communication. Optics Express, 2011, vol. 19, no. 2, p. 482-492. DOI: 10.1364/OE.19.000482

[4] GAO, X., HUANG, S., ZHOU, J., et al. Generating, multiplexing/ demultiplexing and receiving the orbital angular momentum of radio frequency signals using an optical true time delay unit. Journal of Optics, 2013, vol. 15, no. 10, p. 1-6. DOI: $10.1088 / 2040-8978 / 15 / 10 / 105401$

[5] HUANG, H., XIE, G., YAN, Y., et al. 100 Tbit/s free-space data link enabled by three-dimensional multiplexing of orbital angular momentum, polarization, and wavelength. Optics Letters, 2014 vol. 39, no. 2, p. 197-200. DOI: 10.1364/OL.39.000197

[6] GAO, X., HUANG, S., WEI, Y., et al. An orbital angular momentum radio communication system optimized by intensity controlled masks effectively: Theoretical design and experimental verification. Applied Physics Letters, 2014, vol. 105, no. 24, p. 1-5. DOI: 10.1063/1.4904090

[7] REN, Y., WANG, Z., XIE, G., et al. Free-space optical communications using orbital-angular-momentum multiplexing combined with MIMO-based spatial multiplexing. Optics Letters, 2015 , vol. 40, no. 18 , p. 4210-4213. DOI: 10.1364/OL.40.004210

[8] CAI, B. G., LI, Y. B., JIANG, W. X., et al. Generation of spatial Bessel beams using holographic metasurface. Optics Express, 2015, vol. 23, no. 6, p. 7593-7601. DOI: 10.1364/OE.23.007593

[9] ZHANG, C., MA, L. Millimetre wave with rotational orbital angular momentum. Scientific Reports, 2016, vol. 6, no. 3192, p. 1-8. DOI: $10.1038 /$ srep31921

[10] TAMBURINI, F., MARI, E., SPONSELLI, A., et al. Encoding many channels in the same frequency through radio vorticity: First experimental test. New Journal of Physics, 2012, vol. 14, p. 1-17. DOI: $10.1088 / 1367-2630 / 14 / 3 / 033001$

[11] CHENG, L., HONG, W., HAO, Z. C. Generation of electromagnetic waves with arbitrary orbital angular momentum modes. Scientific Reports, 2014, vol. 4, no. 4817, p. 1-5. DOI: 10.1038/srep04814

[12] MOHAMMADI, S. M., DALDORFF, L. K. S., BERGMAN, J. E. $\mathrm{S}$., et al. Orbital angular momentum in radio-A system study. IEEE Transactions on Antennas and Propagation, 2010, vol. 58, no. 2, p. 565-572. DOI: 10.1109/TAP.2009.2037701

[13] MOHAMMADI, S. M., DALDORFF, L. K. S., FOROZESH, K., et al. Orbital angular momentum in radio: Measurement methods. Radio Science, 2010, vol. 45, no. 4, p. 1-14. DOI: $10.1029 / 2009 R S 004299$
[14] CANO, E., ALLEN, B. Multiple-antenna phase-gradient detection for OAM radio communications. Electronics Letters, 2015 , vol. 51, no. 9, p. 724-725. DOI: 10.1049/el.2015.0435

[15] CHEN, J., LIANG, X., HE, C., et al. High-sensitivity OAM phase gradient detection based on time-modulated harmonic characteristic analysis. Electronics Letters, 2017, vol. 53, no. 12, p. $812-814$. DOI: $10.1049 / \mathrm{el} .2016 .4689$

[16] XIE, M., GAO, X., ZHAO, M., et al. Mode measurement of a dual-mode radio frequency orbital angular momentum beam by circular phase gradient method. IEEE Antennas and Wireless Propagation Letters, 2016, vol. 16, p. 1143-1146. DOI: 10.1109/lawp.2016.2624737

[17] GUO, G. R., HU, W. D., DU, X. Y. Electromagnetic vortex based radar target imaging. Journal of National University of Defense Technology, 2013, vol. 35, no. 6, p. 71-76. (in Chinese) DOI: 10.3969/j.issn.1001-2486.2013.06.013

[18] LIU, K., CHENG, Y., YANG, Z., et al. Orbital-angularmomentum-based electromagnetic vortex imaging. IEEE Antennas and Wireless Propagation Letters, 2015, vol. 14, p. 711-714. DOI: 10.1109/lawp.2014.2376970

[19] YUAN, T., WANG, H., QIN, Y., et al. Electromagnetic vortex imaging using uniform concentric circular arrays. IEEE Antennas and Wireless Propagation Letters, 2016, vol. 15, p. 1024-1027. DOI: 10.1109/LAWP.2015.2490169

[20] GE, X., ZI, R., XIONG, X., et al. Millimeter wave communications with OAM-SM scheme for future mobile networks. IEEE Journal on Selected Areas in Communications, 2016, vol. 35 , no. 9, p. 2163-2177. DOI: 10.1109/JSAC.2017.2720238

[21] HU, T., WANG, Y., LIAO, X., et al. OFDM-OAM modulation for future wireless communications. IEEE Access, 2019, vol. 7 p. 59114-59125. DOI: 10.1109/ACCESS.2019.2915035

[22] TAVIK, G. C., HILTERBRICK, C. L., EVINS, J. B., et al. The advanced multifunction RF concept. IEEE Transactions on Microwave Theory and Techniques, 2005, vol. 53, no. 3, p. 1009-1020. DOI: 10.1109/tmtt.2005.843485

[23] BLUNT, S. D., MOKOLE, E. L. Overview of radar waveform diversity. IEEE Aerospace and Electronic Systems Magazine, 2016, vol. 31, no. 11, p. 2-40. DOI: 10.1109/MAES.2016.160071

[24] LIU, F., ZHOU, L., MASOUROS, C., et al. Towards dualfunctional radar-communication systems: Optimal waveform design. IEEE Transactions on Signal Processing, 2018, vol. 66, no. 16 , p. 4264-4279. DOI: 10.1109/TSP.2018.2847648

[25] WANG, Z., LIAO, G., YANG, Z. Space-frequency modulation radar-communication and mismatched filtering. IEEE Access, 2018 , vol. 6, p. 24837-24845. DOI: 10.1109/ACCESS.2018.2829731

[26] LUO, Y., ZHANG, J. A., HUANG, X., et al. Optimization and quantization of multibeam beamforming vector for joint communication and radio sensing. IEEE Transactions on Communications, 2019, vol. 67, no. 9, p. 6468-6482. DOI: 10.1109/TCOMM.2019.2923627

[27] ZHANG, J. A., HUANG, X., GUO, Y. J., et al. Multibeam for joint communication and sensing using steerable analog antenna arrays. IEEE Transactions on Vehicular Technology, 2018, vol. 68 , no. 1, p. 671-685. DOI: 10.1109/TVT.2018.2883796

[28] DALY, M. P., BERNHARD, J. T. Directional modulation technique for phased arrays. IEEE Transactions on Antennas and Propagation, 2009, vol. 57, no. 9, p. 2633-2640. DOI: 10.1109/TAP.2009.2027047

[29] HONG, T., SONG, M. Z., LIU, Y. Dual-beam directional modulation technique for physical-layer secure communication. IEEE Antennas and Wireless Propagation Letters, 2011, vol. 10 , p. 1417-1420. DOI: 10.1109/LAWP.2011.2178384

[30] DING, Y., FUSCO, V. F. A vector approach for the analysis and synthesis of directional modulation transmitters. IEEE 
Transactions on Antennas and Propagation, 2014, vol. 62, no. 1, p. 361-370. DOI: 10.1109/tap.2013.2287001

[31] DING, Y., FUSCO, V. Orthogonal vector approach for synthesis of multi-beam directional modulation transmitters. IEEE Antennas and Wireless Propagation Letters, 2015, vol. 14, p. 1330-1333. DOI: 10.1109/LAWP.2015.2404818

[32] FOUDA, R. M., BAUM, T. C., GHORBANI, K. Quasi-orbital angular momentum (Q-OAM) generated by quasi-circular array antenna (QCA). Scientific Reports, 2018, vol. 8, no. 1, p. 1-11. DOI: $10.1038 / \mathrm{s} 41598-018-26733-6$

[33] THIDÉ, B., THEN, H., SJOHOLM, J., et al. Utilization of photon orbital angular momentum in the low-frequency radio domain. Physical Review Letters, 2007, vol. 99, no. 8, p. 1-4. DOI: 10.1103/ PhysRevLett.99.087701

[34] GONG, Y., WANG, R., DENG, Y., et al. Generation and transmission of OAM-carrying vortex beams using circular antenna array. IEEE Transactions on Antennas and Propagation, 2017, vol. 65, no. 6, p. 2940-2949. DOI: 10.1109/TAP.2017.2695526

[35] TRICHILI, A., PARK, K., ZGHAL M., et al. Communicating using spatial mode multiplexing: Potentials, challenges and perspectives. IEEE Communications Surveys \& Tutorials, 2019, vol. 21, no. 4, p. 3175-3203. DOI: 10.1109/COMST.2019.2915981

\section{About the Authors ...}

Changju ZHU was born in Chuzhou, China. He is currently pursuing the doctor degree in Communication and Information Engineering in Nanjing University of Aeronautics \& Astronautics (NUAA). His research interests include directional modulation (DM) technology, wireless localization technology and signal design.

Maozhong SONG (corresponding author) was born in Shexian, China. He is a Professor in the College of Electronic \& Information Engineering, NUAA. His current research interests include DM technology, signal design, satellite navigation signal processing, wireless localization technology, controlling techniques and communication measuring.

Xiaoyu DANG was born in Wuxi, China. He is a Professor in the College of Electronic \& Information Engineering, NUAA. His current research interests include satellite communications and communication signal processing. 